\title{
Optimization of workcell layouts in mixed-model assembly line environment
}

\author{
Erica Klampfl · Oleg Gusikhin · Giuseppe Rossi
}

\section{Erratum to: Int J Flex Manuf Syst DOI 10.1007/s10696-006-9029-6}

This biographical information was omitted from the original article:

G. Rossi is currently the Technical Leader of the Supply Chain Management Research group at the Ford Research and Innovation Center in Dearborn, Michigan. He holds a Ph.D. in Theoretical Physics from the University of California at San Diego (1983) and an MBA from the University of Michigan, Ann Arbor (2002). Over the course of his career Mr. Rossi has been active in several areas of Theoretical and Computational Physics, Material Science and Operations Research both in academia and in industry. He has authored over 50 refereed publications and holds six US patents.

The online version of the original article can be found under doi:10.1007/s10696-006-9029-6.

E. Klampfl $(\bowtie) \cdot$ O. Gusikhin · G. Rossi

Ford Research and Advanced Engineering, Supply Chain Management Research Group,

Dearborn, MI, USA

e-mail: eklampfl@ford.com 